\title{
Annex IV: Expert Reviewers of the IPCC Special Report on the Oceans and Cryosphere in a Changing Climate
}


ACKERMANN, Thomas

University of Applied Sciences

Germany

\section{ADESALU, TAOFIKAT}

University of Lagos

Nigeria

\section{ADUSUMILLI, Susheel}

Scripps Institution of Oceanography

USA

\section{ADVANI, Nikhil}

World Wildlife Fund

USA

AESCHBACH (NÉE VOLLWEILER), Nicole Heidelberg University

Germany

AGGARWAL, Anubha

Delhi Technological University

India

\section{AHMADUN, Fakhru'L-Razi}

National Defense University of Malaysia

Malaysia

AHMED, Essam Hassan Mohamed

Climate Change and Sustainable

Development Expert

USA

\section{ALAKKAT, Unnikrishnan}

National Institute of Oceanogrphy

India

\section{ALAVA, Juan Jose \\ University of British Columbia \\ Canada}

\section{ALEGRIA, Andrés \\ IPCC WGII TSU}

Alfred Wegener Institute

Germany/Honduras

\section{ALFREDSEN, Knut}

Norwegian University of Science

and Technology

Norway

\section{ALI, Meer}

Florida State University

India
ALI, Syed Hammad

Glacier Monitoring and Research Center

Water and Power Development Authority

Pakistan

\section{ALLAN, Richard \\ University of Reading \\ United Kingdom}

\section{ALLEN, Simon}

University of Zurich

Switzerland

\section{ALLEY, Richard B.}

Pennsylvania State University

USA

ALPERT, Alice

US Department of State

USA

ANDRADE-VELAZQUEZ, Mercedes

Science and Technology Agency-

Global Change and Sustainability

Center in the Southeast

Mexico

\section{ANDRELLO, Marco}

Institut de Recherche pour le Développement

Canada

\section{ANDRESEN, Camilla Snowman}

Geological Survey of Denmark and Greenland

Denmark

ANICAMA DIAZ, Jahir

Pontificia Universidad Católica de Chile

Chile

ANORUO, Chukwuma

IMO State University Owerri

Nigeria

ANTIA, Effiom Edem

University Of Calabar

Nigeria

APPAD00, Chandani

University of Mauritius

Mauritius

ARBLASTER, Julie

Monash University

Australia
ARELLANO-TORRES, Elsa

Universidad Nacional Autónoma de México

Mexico

ARENSON, Lukas

BGC Engineering Inc./University of Manitoba

Canada

ARTHERN, Robert

British Antarctic Survey

United Kingdom

ATWOOD, Trisha

Utah State University

USA

AYALA, Alvaro

Centre for Advanced Studies in Arid Zones

Chile

AYYUB, Bilal

University of Maryland

USA

AZIZ, Danyal

Global Change Impact Studies Center

Pakistan

BABIKER, Mustafa

Saudi Aramco

Saudi Arabia

BAHRI, Tarub

Food and Agriculture Organization

Italy

BALAH, Belkacem

Département d'Hydraulique

Algeria

BALLINGER, Thomas

Texas State University

USA

BAMBER, Jonathan

University of Bristol

United Kingdom

BARANGE, Manuel

Food and Agriculture Organization

Italy

BARLETTA, Valentina R.

DTU Space

Denmark 
BARLOW, Natasha

University of Leeds

United Kingdom

BARR, lestyn

Manchester Metropolitan University

United Kingdom

BARRAZA, Francisco

Center for Climate and Resilience Research Chile

\section{BARRETT, Ko}

IPCC Vice Chair

National Oceanic and Atmospheric

Administration

USA

\section{BARRETT, Peter}

Victoria University of Wellington

New Zealand

BARTLETT, Jesamine

University of Birmingham

United Kingdom

BARTSCH, Annett

Austrian Polar Research Institute

Austria

BARTSCH, Inka

Alfred Wegener Institute

Germany

BASANTES-SERRANO, Rubén

Escuela Politécnica Nacional

Ecuador

BAUMANN, Sabine

TUM

Germany

BEALL, Jacques

Surfrider Foundation Europe

France

BEDNARSEK, Nina

Southern California Coastal

Waters Research Project

USA

BEEVER, Erik

Montana State University and

U.S. Geological Survey

USA
BEHZAD, Layeghi

I.R. of Iran Meteorological Organization Iran

BELL, Robert

National Institute of Water

\& Atmospheric Research

New Zealand

BENKEBLIA, Noureddine

University of the West Indies

Jamaica

BENSON, Nsikak

Covenant University

Nigeria

BERNHARD, Luzi

Swiss Federal Research Institute WSL

Switzerland

\section{BERTHIER, Etienne \\ LEGOS-CNRS \\ France}

BESTER, Marthan

University of Pretoria

South Africa

BEVERIDGE, Malcolm

University of Stirling

United Kingdom

BEYLICH, Achim A.

Geomorphological Field Laboratory

Norway

BHUTIYANI, Mahendra

Defence R \& D Organization

India

BIDDLE, Louise

University of Gothenburg

Sweden

BISHOP-WILLIAMS, Katherine

University of Guelph

Canada

BLARD, Pierre-Henri

CRPG, CNRS, Nancy France and Laboratoire de Glaciologie ULB Bruxelles

France

BLOCKLEY, Ed

Met Office Hadley Centre

United Kingdom
BOCK, Christian

Alfred Wegener Institut

Germany

BODNER, Abigail

Brown University

USA

BOESCH, Donald

University of Maryland Center

for Environmental Sciences

USA

BOLCH, Tobias

University of Zurich

Germany

BONALDO, Davide

Institute of Marine Sciences,

National Research Council

Italy

BÖRGEL, Florian

Insitute for Baltic Sea Research

Germany

BORRAS-CHAVEZ, Renato

Pontificia Universidad Católica de Chile-Center of Applied Ecology and Sustainability

Chile

BOUCHARD, Frédéric

Géosciences Paris Sud

France

BOUWER, Laurens

GERICS

Germany

BOX, Jason

Geological Survey of Denmark and Greenland

Denmark

BRANDT, Angelika

Senckenberg Research Institute

and Natural History Museum

Germany

BRASIER, Madeleine

University of Tasmania

Australia

BRENT, Kerryn

University of Tasmania

Australia 


\section{BRÖDER, Lisa}

Vrije Universiteit

Netherlands

\section{BRONSTERT, Axel}

University of Potsdam

Germany

\section{BROWN, Ross}

Environment and Climate Change Canada Canada

\section{BRUN, Fanny}

Université Grenoble Alpes

France

\section{BURBA, George}

LI-COR Biosciences/University of Nebraska USA

\section{BURGARD, Clara}

Max Planck Institute for Meteorology

Germany

\section{BURGER, Friedrich Anton}

University of Bern

Switzerland

\section{BURGHELEA, Carmen}

University of Arizona

Romania

BURI, Pascal

University of Alaska Fairbanks

USA

\section{BUTENSCHÖN, Momme}

Centro Euro-Mediterraneo sui

Cambiamenti Climatici

Italy

\section{CAESAR, Levke}

Potsdam Institute of Climate Impact Research Germany

\section{CAI, Rongshuo}

Third Institute of Oceanography, Ministry of Natural Resource

China

CALDERON-AGUILERA, Luis Eduardo Centro de Investigacion Cientifica y de Educacion Superior de Ensenada Mexico
CAMERON, Karen

Aberystwyth University

United Kingdom

CAMPBELL, Karley

University of Bristol

United Kingdom

\section{CAMPBELL, Kristin}

Institute for Governance and

Sustainable Development

USA

CAMPBELL, Seth

University of Maine

USA

CAPOBIANCO, Michele

CAPOBIANCO

Italy

\section{CARL-FRIEDRICH, Schleussner}

Climate Analytics

Germany

\section{CARMAGNOLA, Carlo}

Météo-France - CNRS, CNRM,

Centre d'Études de la Neige

France

CARRASCO, Jorge

Universidad de Magallanes

Chile

CARRIVICK, Jonathan

University of Leeds

United Kingdom

\section{CARTER, Peter}

Climate Emergency Institute

Canada

CASADO, Mathieu

Alfred Wegener Institute and APECS

Germany

CASEY, Michael

Germany

CASTANEDA MENA, Fátima Castaneda

Galileo University

Guatemala

CASTANEDA, Fátima

Galileo University

Guatemala
CASTRUCCI, Luca

Pacific Northwest National Laboratory USA

\section{CASWELL, Bryony}

University of Hull

United Kingdom

CAVAN, Emma

University of Tasmania

Australia

CEARRETA, Alejandro

Universidad del País Vasco

Spain

CHAMBERS, robert

Institute of Development Studies

United Kingdom

CHAN, Kim Lian

Unversiti Malaysia SABAH

Malaysia

CHANDRA, Alvin

Department of Environment and Science Australia

CHAPIN, F Stuart

University of Alaska Fairbanks

USA

CHARALAMPIDIS, Charalampos

Bavarian Academy of Sciences and Humanities Germany

CHATURVEDI, Sanjay

Panjab University

India

CHEN, Wenting

Norwegian Institute for Water Research Norway

CHEN, Xingrong

National Marine Environmental

Forecasting Center

China

CHENG, Lijing

Chinese Academy of Sciences

China

CHEUNG, Anson

Brown University

USA 
CHEVALLIER, Matthieu

Météo France

France

\section{CHOWN, Steven}

Monash University and Scientific Committee on Antarctic Research

Australia

CHRISTENSEN, Torben $\mathbf{R}$.

Lund University/Aarhus University

Sweden

\section{CHRISTIANEN, Susan \\ Extreme Design Lab \\ Iceland}

\section{CHUNG CHAI TSANG, Jean Bertrand}

SSR Medical College

Mauritius

\section{CHUNG, Ik Kyo}

Pusan National University

Republic of Korea

\section{CHURCH, John}

University of New South Wales

Australia

\section{CHUST, Guillem \\ AZTI \\ Spain \\ CIPOLLINI, Paolo \\ United Kingdom \\ CLARK, Peter \\ Oregon State University \\ USA}

Telespazio VEGA UK for ESA Climate Office

CLARKE, William

Independent Researcher

Australia

CLAY, Jacinta

Brown University

USA

\section{CLOSSET, Ivia}

University of California Santa Barbara

USA

COBB, Kim

Georgia Tech

USA
COGLEY, J. Graham

Trent University

Canada

COLGAN, William

Geological Survey of Denmark and Greenland

Denmark

COLLEONI, Florence

Istituto Nazionale di Oceanografia

e Geofisica Sperimentale

Italy

COLLINS, Matthew

University of Exeter

United Kingdom

COMTE, Adrien

Université de Brest, IUEM

France

CONDE, Cecilia

National Autonomous University of Mexico

Mexico

CONSTABLE, Andrew

Australian Antarctic Division

Australia

COOK, Joseph

University of Sheffield

United Kingdom

COOLEY, Sarah

Ocean Conservancy

USA

CORNELIUS, Stephen

World Wildlife Federation

United Kingdom

COUGNON, Eva

University of Tasmania

Australia

CRAIG, Marlies

IPCC WGII TSU

University of KwaZulu-Natal

South Africa

CRAMER, Wolfgang

CNRS-IMBE

France

CRAWFORD, Alex

College of Wooster

USA
CRISE, Alessandro

OGS

Italy

CROOKALL, David

UCA

France

CUDENNEC, Christophe

Agrocampus Ouest

France

CUI, Peng

Chinese Academy of Sciences

China

CUNNINGHAM, Sari

University of Oslo

Norway

CVETKOVSKA, Marina

University of Ottawa

Canada

CZYZOWSKA-WISNIEWSKI, Elzbieta

University of Arizona, Pima Community College USA

DADA, Olusegun A.

Federal University of Technology

Nigeria

DAGSSON WALDHAUSEROVA, Pavla

Agricultural University of Iceland

Iceland

DALEI, Narendra

University of Petroleum and Energy Studies India

DANGENDORF, Sönke

University of Siegen

Germany

DANIELA, Coswig Kalikoski

Food and Agriculture Organization Italy

DANSEREAU, Véronique

Nansen Environmental and Remote Sensing Center

Norway

DARMARAKI, Sofia

Meteo France

France 


\section{DAS, Supriyo \\ Presidency University \\ India \\ DAVIES, Kirsten \\ Macquarie University \\ Australia \\ DAWSON, Jackie \\ University of Ottawa \\ Canada}

\section{DAX, Thomas}

Federal Instittue for Less-fevoured and Mountainous Areas

Austria

\section{DE FONTAUBERT, Charlotte \\ The World Bank \\ USA}

DE RAMON N'YEURT, Antoine University of the South Pacific Fiji

DEHECQ, Amaury

California Institute of Technology USA

\section{DEISSENBERG, Christophe \\ Aix-Marseille University Luxembourg}

DEMIROGLU, Osman Cenk Umeå University

Sweden

\author{
DENG, Haijun \\ Fujian Normal University \\ China
}

DENIS ALLEMAND, Denis

Centre Scientifique de Monaco Monaco

\section{DESCAMPS, Sebastien Norwegian Polar institute Norway}

\section{MAURO, Biagio} University of Milano-Bicocca Italy

DIAZ MOREJON, Cristobal Felix Ministry of Science, Technology and the Environment Cuba
DIIWU, John

Government of Alberta

Canada

DIOP, E. Salif

Academy of Sciences

Senegal

DOCQUIER, David

Université Catholique de Louvain

Belgium

\section{DOHERTY, Sarah}

University of Washington

USA

\section{DOLK, Michaela}

Swiss Re

USA

\section{DOVEY, Liz}

Australian National University

Australia

\section{Dow, Christine}

University of Waterloo

Canada

DREYFUS, Gabrielle

Institute for Governance and

Sustainable Development

USA

DRIJFHOUT, Sybren

Royal Netherlands Meteorological Institute Netherlands

\section{DUMONT, Marie}

Meteo-France CNRS, CNRM/CEN

France

DUPAR, Mairi

Overseas Development Institute

United Kingdom

\section{DURGADO0, Jonathan}

GEOMAR Helmholtz Centre

for Ocean Research Kiel

Germany

\section{DUVIVIER, Alice}

National Center for Atmospheric Research USA

\section{ECHEVESTE, Pedro}

Instituto de Ciencias Naturales

Alexander von Humboldt

Chile

EDDEBBAR, Yassir

Scripps Institution of Oceanography

USA

EDDY, Tyler

University of British Columbia

Canada

EDWARDS, Charity

University of Melbourne

Australia

EDWARDS, Martin

Sir Alister Hardy Foundation for Ocean Science United Kingdom

EDWARDS, Tamsin

King's College London United Kingdom

EERKES-MEDRANO, Laura

University of Victoria

Canada

EGGERMONT, Hilde

Belgian Science Policy Office

Belgium

EHRENFELS, Benedikt

EAWAG \& ETH Zurich

Switzerland

EINALI, Abbas

PMO

Iran

ELING, Lukas

Victoria University of Wellington

New Zealand

ELLIFF, Carla

Universidade Federal da Bahia

Brazil

ELOKA-EBOKA, Andrew University of KwaZulu-Natal South Africa 
ENGELBRECHT, Francois

University of the Witwatersrand

South Africa

ENGLAND, Mark

Columbia University

United Kingdom

\section{ENGSTRÖM, Erik}

Swedish Meteorological and

Hydrological Institute

Sweden

EPSTEIN, Howard

University of Virginia

USA

ERLANIA, Erlania

Center for Fisheries Research

Indonesia

ERNANI DA SILVA, Carolina

TU Delft

Netherlands

ERWIN, Rottler

University of Potdam

Germany

FARIA, Sérgio Henrique

Basque Centre for Climate Change

Spain

FARINOTTI, Daniel

VAW ETH Zurich / WSL Birmensdorf

Switzerland

FATORIC, Sandra

North Carolina State University

USA

\section{FEELY, Richard}

National Oceanographic and

Atmospheric Administration

USA

FEHRENBACHER, Jennifer

Oregon State University

USA

FELDMAN, Daniel

Lawrence Berkeley National Laboratory USA
FELIKSON, Denis

National Aeronautics and Space Administration USA

\author{
FERNANDEZ PEREZ, Fiz \\ CSIC \\ Spain
}

FERNANDINO, Gerson

Red ProPlayas

Brazil

FETTWEIS, Xavier

University of Liège

Belgium

FIAMMA, Straneo

Scripps/University of California San Diego

USA

FIERZ, Charles

WSL Institute for Snow and

Avalanche Research SLF

Switzerland

FIRING, Yvonne

National Oceanography Centre

United Kingdom

FISCHLIN, Andreas

IPCC WGII Vice Chair

ETH Zurich

Switzerland

FIZ, Fernandez

CSIC

Spain

FLEMING, Sean

Oregon State University/ University of British Columbia/ White Rabbit R\&D LLC USA

\section{FLOWERS, Gwenn}

Simon Fraser University

Canada

FOGGIN, Marc

University of Central Asia

Kyrgyzstan

FOGWILL, Christopher

Keele University

United Kingdom
FORD, James

McGill University

Canada

FORD, Victoria

Texas A\&M University

USA

FORSBERG, Rene

Technical University of Denmark

Denmark

FÖRSTER, Kristian

Leibniz Universität Hannover

Germany

FOURNIER, Aimé

MIT

USA

FOX-KEMPER, Baylor

Brown University

USA

FRA.PALEO, Urbano

University of Extremadura

Spain

FRAJKA-WILLIAMS, Eleanor

National Oceanography Centre

United Kingdom

FRANS-JAN, Parmentier

University of Oslo

Norway

FREPPAZ, Michele

Università degli Studi di Torino Italy

FROELICHER, Thomas

University of Bern

Switzerland

FUGLESTVEDT, Jan

Centre for International Climate and Environmental Research

Norway

FÜSSEL, Hans-Martin European Environment Agency Denmark

GAGLIARDINI, Olivier Université Grenoble Alpes

France 
GAGNE, Karine

University of Guelph

Canada

GALBÁN-MALAGÓN, Cristóbal

Universidad Andrés Bello

Chile

\section{GALEN MCKINLEY, Galen}

Lamont Doherty Earth Observatory/

Columbia University

USA

GAN, Thian Yew

University of Alberta

Canada

GAO, Jing

Chinese Academy of Sciences

China

GARCIA-REYES, Marisol

Farallon Institute for Advanced

Ecosystem Research

USA

GARELICK, Sloane

Brown University

USA

GARNER, Andra

Rutgers University

USA

GARZA-GIL, M. Dolores

University of Vigo

Spain

GATTUSO, Jean-Pierre

CNRS-Sorbonne Université-Iddri

France

GELDSETZER, Torsten

University of Calgary

Canada

GERLAND, Sebastian

Norwegian Polar Institute

Norway

GIDDY, Isabelle Sindiswa

University Of Cape Town

South Africa

GIOLI, Giovanna

University of Edinburgh

United Kingdom
GLASER, Paul

University of Minnesota

USA

GOETZ, Scott

Northern Arizona University

USA

GOHEER, Muhammad

GCISC

Pakistan

GOLLEDGE, Nicholas

Victoria University of Wellington

New Zealand

GONG, Crystal

University of Alberta

Canada

GONZALEZ, Patrick

University of California

USA

GOUTTEVIN, Isabelle

Météo-France

France

GREGOIRE, Marilaure

MAST -ULiege

Belgium

GREGORY, Jonathan

University of Reading

United Kingdom

GREMION, Gwenaëlle

Université du Québec à Rimouski

Canada

GRIBBLE, Matthew

Emory University

USA

GROSSE, Guido

Alfred Wegener Institute

Germany

GRUBER, Nicolas

ETH Zürich

Switzerland

GRUBER, Stephan

Carleton University

Canada
GUARINO, Maria Vittoria

British Antarctic Survey

United Kingdom

GUILLAUME, Anne

Météo et Climat

France

GUILLOUX, Bleuenn Gaëlle

Cluster of Excellence the Future Ocean

Germany

GUILYARDI, Eric

LOCEAN/IPSL CNRS

France

GUNDERSEN, Hege

Norwegian Institute for Water Research

Norway

GUPTA, Mukesh

Institut de Ciencies del Mar

Spain

HAASNOOT, Marjolijn

Delft University of Technology

Netherlands

HAEBERLI, Wilfried

University of Zurich

Switzerland

HAINE, Thomas

Johns Hopkins University

USA

HALL-SPENCER, Jason

University of Plymouth/University of Tsukuba

United Kingdom/Japan

HAMILTON, Lawrence

University of New Hampshire

USA

HAN, In-Seong

National Institute of Fisheries Science

Republic of Korea

HANSMAN, Roberta

International Atomic Energy Agency

France

HAQUE, Md Enamul

University of Genova

Italy 
HARDMAN-MOUNTFORD, Nicholas

Commonwealth Secretariat

United Kingdom

HASHMI, Danial

WAPDA

Pakistan

HAWARD, Marcus

Unversity of Tasmania

Australia

HAYASHI, Kentaro

National Agriculture and Food

Research Organization

Japan

HAYASHI, Masaki

University of Calgary

Canada

\section{HAYMAN, Garry}

Centre for Ecology \& Hydrology

United Kingdom

HELENE, Frigstad

Norwegian Institute for Water Research

Norway

\section{HERNÁNDEZ, Armand \\ ICTJA-CSIC \\ Spain}

HEWITT, Helene

Met Office

United Kingdom

HEYD, Thomas

University of Victoria

Canada

HIDEKI, Kanamaru

Food and Agriculture Organization

Thailand

\section{HIERONYMUS, Magnus}

Swedish Meteorological and

Hydrological Institute

Sweden

HIRABAYASHI, Yukiko

Shibaura Institute of Technology

Japan

HJøLLO, Solfrid Sætre

Institute of Marine Research

Norway
HJORT, Jan

University of Oulu

Finland

HOCK, Regine

University of Alaska Fairbanks

USA

HOENISCH, Baerbel

University

USA

HOFFMANN, Dirk

Bolivian Mountain Institute

Germany

HOFSTEDE, Jacobus

Schleswig-Holstein Ministry for

Energy, Agriculture, Environment,

Nature and Digitization

Germany

HOLDING, Johnna

Aarhus University

Denmark

HOPWOOD, Mark

Geomar Helmholtz Centre for Ocean Research Kiel

Germany

HOVLAND, Martin

University of Tromsø

Norway

HOWARD, William

Australian National University

Australia

HU, Zeng-Zhen

National Oceanographic and

Atmospheric Administration

USA

HUANG, Ping

Chinese Academy of Sciences

China

HUDSON, Thomas

British Antarctic Survey

United Kingdom

HUETTMANN, Falk

University of Alaska Fairbanks

USA
HUGGEL, Christian

University of Zurich

Switzerland

HUNT, George

University of Washington

USA

HUNTER, Nina

University of KwaZulu-Natal

South Africa

HUNTINGTON, Henry

Huntington Consulting

USA

HURLBERT, Margot

University of Regina

Canada

HUSS, Matthias

ETH Zürich

Switzerland

IBRAHIM, Zelina

Universiti Putra Malaysia

Malaysia

IMMERZEEL, Walter

Utrecht University

Netherlands

INSAROV, GRIGORY

Russian Academy of Sciences

Russian Federation

ISLAM, Akm Saiful

Bangladesh University of

Engineering and Technology

Bangladesh

ITKIN, Polona

Nansen Environmental and

Remote Sensing Center

Norway

ITO, Shin-Ichi

University of Tokyo

Japan

JACKSON, Lagipoiva Cherelle National University of Samoa

Samoa

JACKSON, Laura

Met Office Hadley Centre

United Kingdom 
JAFARI, Mostafa

National Macro Plan on Climate

Change Research/RIFR

Iran

JAHN, Alexandra

University of Colorado Boulder

USA

JAMIE, Shutler

University of Exeter

United Kingdom

JANSSEN, David

University of Bern

Switzerland

\section{JENNINGS, Keith}

University of Nevada, Reno and

The Desert Research Institute

USA

\section{JEWETT, Elizabeth}

National Oceanographic and

Atmospheric Administration

USA

JIE, Liu

University of Bergen

Norway

\section{JOCHUMSEN, Kerstin}

Federal Maritime and Hydrographic Agency

Germany

\section{JOHN, Emeka}

Georg-August-Universität Göttingen

Germany

\section{JOHN, Jasmin}

National Oceanographic and

Atmospheric Administration

USA

\section{JOHNSON, Joanne}

British Antarctic Survey

United Kingdom

\section{JÖNSSON, Anette}

Swedish Meteorological and

Hydrological Institute

Sweden

JOOS, Fortunat

University of Bern

Switzerland
JOSEY, Simon

National Oceanography Centre

United Kingdom

JOURDAIN, Nicolas

IGE, CNRS/Univ. Grenoble-Alpes

France

JOUZEL, Jean

CEA

France

JRRAR, Amna

Independent Researcher

Jordan

JUAN IGNACIO, López Moreno

CSIC

Spain

KÄÄB, Andreas

University of Oslo

Norway

KADIBI, Khadija

National Meteorological Direction

Morocco

KALEN, Ola

Swedish Meteorological

and Hydrological Institute

Sweden

KALÉN, Ola

Swedish Meteorological

and Hydrological Institute

Sweden

KANDASAMY, Kathiresan

Annamalai University

India

KANTH, Malin

Governmental Agency

Sweden

KAPNICK, Sarah

National Oceanographic and

Atmospheric Administration

USA

KAPSENBERG, Lydia

CSIC Institute of Marine Sciences Spain
KASER, Georg

University of Innsbruck

Austria

KATSUMATA, Katsuro

JAMSTEC

Japan

KAWAMIYA, Michio

Japan Agency for Marine-Earth

Science and Technology

Japan

KENNEDY, Hilary

Bangor University

United Kingdom

KENNY, Tiff-Annie

Nereus Program

Canada

KERSTING, Diego

Freie Universität Berlin

Germany

KETTLES, Helen

Department of Conservation

New Zealand

KHAEMBA, Winnie

African Centre for Technology Studies Kenya

KHALEEL, Zammath

Ministry of Environment

Maldives

KHESHGI, Haroon

ExxonMobil

USA

KIESSLING, Wolfgang

Friedrich-Alexander University

Erlangen-Nürnberg

Germany

KIM, Seong-Joong

Korea Polar Research Institute

Republic of Korea

KIM, Sung Yong

Korea Advanced Institute of

Science and Technology

Republic of Korea 
KIMBLE, Melinda

United Nations Foundation

Syracuse University

USA

KING, Matt

University of Tasmania

Australia

KITOH, Akio

Japan Meteorological Business Support Center

Japan

\section{KJELDSEN, Kristian Kjellerup}

Geological Survey of Denmark and Greenland

Denmark

KNIEBUSCH, Madline

Leibniz Institute for Baltic Sea

research Warnemünde

Germany

KOBASHI, Takuro

Renewable Energy Institute

Japan

\section{KOCHTITZKY, William}

University of Maine

USA

KOHNERT, Katrin

GFZ German Research Centre for Geosciences

Germany

KOIVUROVA, Timo

Arctic Centre/University of Lapland

Finland

\section{KONOVALOV, Vladimir \\ Institute of Geography \\ Russian Federation}

\section{KONYA, Keiko}

Japan Agency for Mari-Earth

Science and Technology

Japan

KOPP, Robert

Rutgers University

USA

KOSZALKA, Inga

GEOMAR Helmholtz Centre for Ocean

Research Kiel \& Kiel University

Germany
KOTLARSKI, Sven

Federal Office of Meteorology

and Climatology MeteoSwiss

Switzerland

KOUL, Vimal

Universität hamburg

Germany

KOURANTIDOU, Melina

University of Southern Denmark

Denmark

KOVEN, Charles

Lawrence Berkeley National Lab USA

KRAUSE-JENSEN, Dorte

Aarhus University

Denmark

\section{KRINNER, Gerhard \\ IGE/CNRS \\ France}

KROGLUND, Marianne

Norwegian Environment Agency/Arctic

Monitoring and Assessment Programme

Norway

KRUEMMEL, Eva

ScienTissiME, Inuit Circumpolar Council

Canada

KUDELA, Raphael

University of California Santa Cruz

USA

KUSWARDANI, Anastasia Rita Tisiana Dwi Ministry of Marine Affairs and Fisheries Indonesia

KUWAE, Tomohiro

Port and Airport Research Institute

Japan

\section{KWIATKOWSKI, Lester}

Laboratoire de Météorologie Dynamique, Institut Pierre-Simon Laplace

France

KYZIVAT, Ethan

Brown University

USA
LAGERLOEF, Gary

Earth and Space Research (Retired)

USA

LAM, Steven

University of Guelph

Canada

LANDOLFI, Angela

GEOMAR Helmholtz Centre

for Ocean Research

Germany

LANGE, Benjamin A.

Fisheries and Oceans Canada

Canada

LAVRILLIER, Alexandra

CEARC, OVSQ, Versailles University

France

LAW, Cliff

National Institute of Water \&

Atmospheric Research

New Zealand

LAWRENCE, Judy

Victoria University of Wellington

New Zealand

LAYEGHI, Behzad

IRIMO

Iran

\section{LE BARS, Dewi}

KNMI

Netherlands

LE COZANNET, Goneri

BRGM

France

LE QUERE, Corinne

University of East Anglia

United Kingdom

LE TRAON, Pierre Yves

Mercator Ocean International

France

LE, Hoang Anh

Vietnam

LECLERC, Boris

Public Institution of the Ministry of Environment France 
LEE-SIM, Lim

Universiti Sains Malaysia

Malaysia

LEE, Brown

University of Leeds

United Kingdom

\section{LEE, Sai Ming \\ Hong Kong Observatory \\ China}

\section{LEE, Suk Hui}

Korea Marine Environment

Management Corporation

Republic of Korea

\section{LEE, WON SANG}

Korea Polar Research Institute

Republic of Korea

LEE, Yoo Kyung

Korea Polar Research Institute

Republic of Korea

LEGG, Sonya

Princeton University

USA
LIAO, Wenjie

Sichuan University

China

LIFANG, Chiang

University of California

USA

LIM, Lee-Sim
Universiti Sains Malaysia
Malaysia

LINDBÄCK, Katrin

Norwegian Polar Insitute

Norway

LING, Frank

University of Tokyo

Japan

LIPKA, Oxana

WWF Russia

Russian Federation

LIU, Jihua

Shandong University

China

LIU, Kexiu

National Marine Data and Information Service

China

LIU, Shiyin

Yunnan University

China

LIVINGSTON, Mary

Fisheries New Zealand, Ministry

for Primary Industries

New Zealand

LEY, Debora

University of Oxford

Guatemala

LI, Hai

Ministry of Natural Resources

China

LI, Xichen

Chinese Academy of Sciences

China

LIANG, Yantao

Chinese Academy of Sciences

China
LLANILLO, Pedro J.

University of Santiago de Chile

Chile

LOCKLEY, Andrew

University College London

United Kingdom

\section{LOKMAN, Kees}

University of British Columbia

Canada

LOMBARDI, Chiara

Italian National Agency for New

Technologies, Energy and Sustainable

Economic Development

Italy
LOPEZ-GASCA, Mariela

Venezuelan Research Institute

Venezuela

LORANTY, Michael

Colgate University

USA

LORENZ, William

University of Southern Queensland

Australia

LORENZONI, Laura

University of South Florida

USA

LOVEJOY, Connie

Université Laval

Canada

LOWTHER, Andrew

Norwegian Polar Institute

Norway

LUBANGO, Louis Mitondo

United Nations Economic

Commission for Africa

Ethiopia

LUCEY, Noelle

Smithsonian Tropical Research Institute Panama

LUENING, Sebastian

Institute for Hydrography, Geoecology and Climate Sciences

Portugal

LUKAS, Sven

Lunds Universitet

Sweden

LUPO, Anthony

University of Missouri

USA

ŁUSZCZUK, Michał

Maria Curie Skłodowska University

Poland

MACCRACKEN, Michael

Climate Institute

USA

MACDONALD, Joanna

Inuit Circumpolar Council Canada

Canada 
MACDONALD, Robie

Fisheries and Oceans Canada

Canada

MADSEN, Kristine Skovgaard

Danish Meteorological Institute

Denmark

\section{MAGNAN, Alexandre \\ IDDRI \\ France}

MAHMOOD, Ali

University of Basrah

Iraq

\section{MAHMUD, Mastura \\ Universiti Kebangsaan Malaysia}

Malaysia

MAJID COOKE, Fadzilah

National University of Malaysia

Malaysia

\section{MALATESTA, Stefano}

University of Milano-Bicocca

Italy

MAŁECKI, Jakub

Adam Mickiewicz University in Poznań

Poland

\section{MANTILLA-MELUK, Hugo \\ Unversidad del Quindío \\ Colombia}

\section{MARBAIX, Philippe}

Université Catholique de Louvain

Belgium

MARCIL, Catherine

Institut des Sciences de la Mer

Canada

\section{MARKUSZEWSKI, Piotr}

Polish Academy of Sciences

Poland

MARTIN-VIDE, Javier

University of Barcelona

Spain

\section{MARTINERIE, Patricia}

Institut des Géosciences de

I'Environnement, CNRS

France

\author{
MARTÍNEZ FONTAINE, Consuelo \\ Université Paris-Saclay \\ France
}

\author{
MARTY, Christoph \\ SLF \\ Switzerland \\ MASSON-DELMOTTE, Valerie \\ IPCC WGII Co-Chair \\ LSCE \\ France
}

MASSONNET, François

Université Catholique de Louvain and

Barcelona Supercomputing Center

Belgium

MATT, King

University of Tasmania

Australia

MATTHEW, Collins

University of Exeter

United Kingdom

MAURITZEN, Cecilie

Norwegian Meteorological Institute Norway

MAXIMILLIAN, Van Wyk de Vries

University of Minnesota

USA

MCCARTHY, Gerard

Maynooth University

Ireland

MCDOWELL, Graham

University of British Columbia

Canada

MCGEE, Jeffrey

University of Tasmania

Australia

MEDINA, Josep Ramon

Universitat Politècnica de València

Spain

MEINANDER, Outi

Finnish Meteorological Institute

Finland

MELET, Angelique

Mercator Ocean International

France
MÉMIN, Anthony

Université Côte d'Azur

France

MENARY, Matthew

Met Office Hadley Centre

United Kingdom

MENDOZA, Marcos

University of Mississippi

USA

MÉNÉGOZ, Martin

Institut des Géosciences de I'Environnement

France

MENGEL, Matthias

Potsdam Institute for Climate Impact Research

Germany

MENVIEL, Laurie

University of New South Wales

Australia

MIDGLEY, Pauline

Independent Consultant

Germany

MIGNOT, Juliette

IRD-IPSL/LOCEAN

France

MILINEVSKY, Gennadi

Taras Shevchenko University of Kyiv Ukraine

MILLS, L. Scott

University of Montana

USA

MILNER, Alexander

University of Birmingham

United Kingdom

MINER, Kimberley

University of Maine

USA

MINTENBECK, Katja

IPCC WGII TSU

Alfred Wegener Institute

Germany

MISHRA, Anil

United Nations Educational,

Scientific and Cultural Organization

France 


\section{MIX, Alan}

Oregon State University

USA

MONCKTON OF BRENCHLEY, Viscount

Science and Public Policy Institute

United Kingdom

\section{MONTPETIT, Benoit}

Environment and Climate Change Canada

Canada

MOORE, Robert

University of British Columbia

Canada

MOORE, Tommy

Northwest Indian Fisheries Commission

USA

\author{
MORA, Carla \\ IGOT \\ Portugal \\ MORAN, Kate \\ University of Victoria \\ Canada

\section{MORELLE, Nathalie} \\ Permanent Secretariat of the \\ Alpine Convention \\ Austria \\ MORIN, Samuel \\ Météo-France - CNRS \\ France

\section{MOSER, Gleyci} \\ Universidade Estadual do Rio de Janeiro \\ Brazil
}

\section{MOTTRAM, Ruth \\ DMI}

Denmark

\section{MOUREY, Jacques}

University Grenoble Alpes/University

Savoie Mont Blanc/CNRS

France

\section{MRAK, Irena}

Environmnetal Protection College

Slovenia

\section{MSADEK, Rym}

CNRS
France

MUELLER, Bennit

Institute of Ocean Sciences

Canada

MÜLLER, Marius

Federal University of Pernambuco

Brazil

MUÑOZ SOBRINO, Castor

Universidade de Vigo

Spain

MURATA, Akihiko

RCGC/JAMSTEC

Japan

MURPHY, Eugene

British Antarctic Survey

United Kingdom

NAM, Sunghyun

Seoul National University

Republic of Korea

NAUELS, Alexander

Climate Analytics

Germany

NAUGHTEN, Kaitlin

British Antarctic Survey

United Kingdom

NDIONE, Jacques-Andre

Centre de Suivi Ecologique

Senegal

NEBDI, Hamid

Chouaïb Doukkali University

Morocco

NELSON, Joanna

LandSea Science

USA

NEOGI, Suvadip

IPCC WGIII TSU

Ahmedabad University

India

NERILIE, Abram

Australian National University

Australia

NICHOLLS, Robert

University of Southampton

United Kingdom
NICOLAI, Maike

IPCC WGII TSU

Alfred Wegener Institute

Germany

NIENOW, Peter

University of Edinburgh

United Kingdom

NIFENECKER, Herve

Sauvons Le Climat

France

NIKAM, Jaee

UN Environment

India

NORTH, Michelle A.

University of KwaZulu-Natal

South Africa

NOTZ, Dirk

Max Planck Institute for Meteorology Germany

NUGRAHA, Adi

Pacific Northwest National Laboratory USA

NÜSSER, Marcus

Heidelberg University

Germany

OAKES, Robert

United Nations University Institute for Environment and Human Security

United Kingdom

OGUTU, Geoffrey Evans Owino

Meteorological Services

Kenya

OKEM, Andrew

IPCC WGII TSU

University of KwaZulu-Natal

South Africa/Nigeria

OLIVA, Frank

Environment and Climate Change Canada

Canada

OLIVA, Marc

University of Barcelona

Spain 


\section{ONINK, Victor}

University of Bern

Switzerland

\section{ONO, Tsuneo}

Japan Fisheries Research and

Education Agency

Japan

ORENSTEIN, Patrick

Brown University

USA

\section{ORLOVE, Ben}

Columbia University

USA

ORR, James

Climate \& Environment Sciences Lab/IPSL, CEA-CNRS-UVSQ

France

OSWALD SPRING, Úrsula UNAM, National Autonomous University of Mexico

Mexico

\section{OTTERSEN, Geir}

Institute of Marine Research

Norway

\section{OTTO SIMONETT, otto}

Zoï Environment Network

Switzerland

OTTO, Jan-Christoph

University of Salzburg

Germany

\section{OUILLON, Sylvain}

Institut de Recherche pour le Développement

France

\author{
OURBAK, Timothée \\ AFD \\ France
}

OVE HOEGH-GULDBERG, Ove

University of Queensland

Australia

OVERDUIN, Pier-Paul

Alfred Wegener Institute

Germany

\section{OZSOY, Burcu}

Istanbul Technical University

Polar Research Center

Turkey

PALMER, Matthew

Met Office Hadley Centre

United Kingdom

PALUPI, Listyati

Airlangga University

Indonesia

PARK, Jinsoon

Korea Maritime and Ocean University

Republic of Korea

PARK, Taehyun

Greenpeace East Asia

Republic of Korea

PATTYN, Frank

Université libre de Bruxelles

Belgium

PAUL, Frank

University of Zurich

Switzerland

PAULI, Harald

Austrian Academy of Sciences, University of Natural Resources and Life Sciences Vienna Austria

PAYNE, Mark

Technical University of Denmark

Denmark

\section{PEARSON, Jenna}

Brown University

USA

PEARSON, Pamela

International Cryosphere Climate Initiative USA

\section{PEBAYLE, Antoine}

Ocean and Climate Platform

France

PELEJERO, Carles

ICREA and Institut de Ciències del Mar, CSIC Spain

\section{PENG, Ge}

North Carolina State University/National Centers for Environmental Information USA

PENTZ, Brian

University of Toronto

Canada

PEREIRA, Christopher

Secretariat of the Convention on Biological Diversity

Canada

PETER, Croot

National University of Ireland Galway Ireland

PETER, Maria

Norwegian University of

Science and Technology

Norway

PETRASEK MACDONALD, Joanna

Inuit Circumpolar Council

Canada

PETRIE, Elizabeth

University of Glasgow

United Kingdom

PETZOLD, Jan

IPCC WGII TSU

Alfred Wegener Institute

Germany

PEZZoLI, Alessandro

Politecnico di Torino e Università di Torino Italy

PFEFFER, Julia

The Australian National University Australia

PIEPENBURG, Dieter

Alfred Wegener Institute

Germany

PIERCE, Ethan

Brown University

USA

PLANTON, Serge

Météo-France, CNRM

France 
POHJOLA, Veijo

Uppsala University

Sweden

POITOU, Jean

Sauvons Le Climat

France

\section{POLOCZANSKA, Elvira S.}

IPCC WGII TSU

Alfred Wegener Institute

Germany/United Kingdom

POPE, James

British Antarctic Survey

United Kingdom

PÖRTNER, Hans-Otto

IPCC WGII Co-Chair

Alfred Wegener Institute

Germany

\section{POULAIN, Florence}

Food and Agriculture Organization

Italy

\section{POUSSIN, Charlotte}

University of Geneva and UNEP GRID

Switzerland

\section{PRINZ, Rainer \\ University of Graz \\ Austria}

\section{QUEIROS, Ana}

Plymouth Marine Laboratory

United Kingdom

\section{QUEIRÓS, José Pedro \\ University of Coimbra \\ Portugal}

\section{RABATEL, Antoine}

University of Grenoble

France

\section{RABE, Benjamin}

Alfred Wegener Institute

Germany

RABEHI, Walid

CTS/ASAL

Algeria

\section{RABOUILLE, Sophie}

CNRS

France
RACAULT, Marie-Fanny

Plymouth Marine Laboratory

United Kingdom

RAHMSTORF, Stefan

Potsdam Institute for Climate Impact Research

Germany

RAJAPAKSHE, Chamara

University of Maryland Baltimore County

Sri Lanka

RAMA, Bardhyl

IPCC WGII TSU

Alfred Wegener Institute

Germany/Kosovo

RAMAGE, Justine

Stockholm University

Sweden

RAMOS, Isabel

SENAMHI

Peru

RANZI, ROBERTO

University of Brescia

Italy

RASHIDIAN, Leila

OASC, IRIMO

Iran

RASSMANN, Jens

University of Liège

Belgium

RASUL, Golam

International Centre for Integrated

Mountain Development

Nepal

REBETEZ, Martine

WSL and University of Neuchatel

Switzerland

RECINOS RIVAS, Beatriz Margarita

University of Bremen

Germany

RECKIEN, Diana

Universiteit Twente

Germany

REESE, Ronja

Potsdam Institute for Climate Impact Research Germany
REID, Keith

Commission for the Conservation

of Antarctic Marine Living Resources

Australia

RENNER, Angelika

Institute of Marine Research

Norway

RENWICK, James

Victorial University of Wellington

New Zealand

REUTEN, Christian

University of Calgary

Canada

RICHTER, Claudio

Alfred Wegener Institute/ University of Bremen

Germany

RICHTER, Nora

Brown University

USA

RIDING, Tim

New Zealand Ministry for the Environment

New Zealand

RIIHELÄ, Aku

Finnish Meteorological Institute

Finland

RIMI, Ruksana

University of Oxford

United Kingdom

RINTOUL, Stephen

Commonwealth Scientific and

Industrial Research Organisation

Australia

RIYAZ, Mahmood

Maldivian Coral Reef Society

Maldives

ROBERTS, Debra

IPCC WGII Co-Chair

EThekwini Municipality

South Africa

ROCKMAN, Marcy

International Council on Monuments and Sites USA

ROHMER, Jeremy

Bureau de Recherches Géologiques et Minières Finland 
ROSE, Tseng

Air Force Institute of Technology

USA

ROSS, Nathan

Ministry of Foreign Affairs and Trade

New Zealand

\section{RUBENSDOTTER, Lena \\ Geological Survey of Norway \\ Norway}

RUIZ, Lucas

Instituto Argentino de Nivología,

Glaciología y Ciencias Ambientales

Argentina

RUMMUKAINEN, Markku

Swedish Meteorological and

Hydrological Institute

Sweden

RUNCIE, John

University of Sydney

Australia

RYABININ, Vladimir

Intergovernmental Oceanographic Commission

France

RYBSKI, Diego

Potsdam Institute for Climate Impact Research

Germany

RYKACZEWSKI, Ryan

University of South Carolina

USA

\section{SAITO, Kazuyuki \\ JAMSTEC \\ Japan}

\section{SAKYA, Andi Eka}

Agency for the Assessment and

Application of Technology

Indonesia

SALA, Hernan Edgardo

Argentine Antarctic Institute

Argentina

SALERNO, Franco

IRSA-CNR

Italy

SALLEE, Jean Baptiste

LOCEAN/CNRS

France
SAMELSON, Roger

Oregon State University

USA

SAMMIE, Buzzard

University College London

United Kingdom

SANDBERG SOERENSEN, Louise

DTU Space

Denmark

SANDER, Sylvia

IAEA-NAEL

France

SANE, Aakash

Brown University

USA

SANZ SANCHEZ, Maria Jose

Basque Centre for Climate Change

Spain

SATO, Kirk

Okinawa Institute of Science and Technology Japan

SATOH, Masaki

University of Tokyo

Japan

SAVOSKUL, Oxana

Sri Lanka

SCHARFFENBERG, Martin

University of Hamburg

Germany

SCHAUWECKER, Simone

University of Geneva

Chile

SCHLEUSSNER, Carl-Friedrich

Climate Analytics

Germany

SCHLOGEL, Romy

ESA Climate Office

United Kingdom

SCHOEMAN, David

University of the Sunshnie Coast

Australia
SCHÖLD, Sofie

Swedish Meteorological and Hydrological Institute

Sweden

SCHRAM, Julie

University of Oregon

USA

SCHRAMA, Ernst

Delft University of Technology

Netherlands

SCHREIBER, Erika

University of Colorado Boulder USA

SCHROEDER, Katrin

CNR ISMAR

Italy

SCHUBACK, Nina

EPFL

Switzerland

SCHULER, Thomas Vikhamar University of Oslo

Norway

SCHWEBEL, Michael

Temple University/The Johns

Hopkins University

USA

\section{SCHWEIZER, Juerg}

WSL Institute for Snow and

Avalanche Research

Switzerland

SEFERIAN, Roland

CNRM (Météo-France/CNRS)

France

SEIBERT, Petra

University of Natural Resources and Life Sciences

Austria

SENSOY, Serhat

Turkish State Meteorological Service Turkey

SERGI, Sara

LOCEAN-IPSL

France 


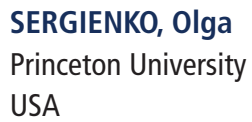

\section{SHIGEMITSU, Masahito}

Japan Agency for Marine-Earth

Science and Technology

Japan

\section{SHRESTHA, Arun}

International Centre for Integrated

Mountain Devlopment

Nepal

\section{SHRESTHA, Maheswor}

Water and Energy Commission Secretariat Nepal

\section{SHUTLER, Jamie \\ University of Exeter \\ United Kingdom}

\section{SILLMANN, Jana}

Centre for International Climate and Environmental Research Norway

\section{SILVESTRE, Elizabeth \\ Universidad Catolica Santo \\ Toribio de Mogrovejo \\ Peru \\ SINGH, Nayanika \\ Ministry of Environment, Forest and Climate Change India \\ SINGH, Shalini \\ University of the South Pacific Fiji \\ SINKLER, Emilie \\ University of Alaska Fairbanks USA}

\section{SIORAK, Nicolas}

Business Alliance for Climate Resilience France

\section{SISWANTO, Siswanto}

The Agency for Meteorology, Climatology, and Geophysics Indonesia
SKEIE, Ragnhild Bieltvedt

Centre for International Climate

and Environmental Research

Norway

\section{SLANGEN, Aimee}

Royal Netherlands Institute for Sea Research Netherlands

\section{SMEDSRUD, Lars \\ University of Bergen \\ Norway \\ SMEED, David \\ National Oceanography Centre \\ United Kingdom}

SMITH, Inga

University of Otago

New Zealand

\section{SMITH, Sharon}

Geological Survey of Canada

Natural Resources Canada

Canada

\section{SMOLYANITSKY, Vasily}

Arctic and Antarctic Research Institute

Russian Federation

\section{SOMMARUGA, Ruben}

University of Innsbruck

Austria

SOUZA, Alejandro

CINVESTAV

Mexico

SOYSA, Ramesh

PELCO Development Consultants Pvt. Ltd.

Sri Lanka

\section{SPANDRE, Pierre}

Institu National de Recherche en Sciences et Technologies de l'Environnement et Agriculture France

SPEER, Elizabeth

Natural Resources Defense Council USA

SPEER, Lisa

Natural Resources Defense Council USA

SPENCER, Thomas

University of Cambridge United Kingdom
SPRING, Aaron

MPI-M

Germany

SROKOSZ, Meric

National Oceanography Centre

United Kingdom

ST. PIERRE, Kyra

University of Alberta

Canada

STENDEL, Martin

Danish Meteorological Institute

Denmark

STENMARK, Aurora

Norwegian Environment Agency

Norway

STOJANOV, Robert

Mendel University in Brno/

European University Institute

Czech Republic

STORCH, Daniela

Alfred Wegener Institute

Germany

STRANEO, Fiamma

Scripps/University of California

USA

STRAUSS, Sarah

University of Wyoming

USA

STRIEGEL, Sandra

University of Bern

Switzerland

STROBACH, Ehud

National Aeronautics and Space Administration USA

STUDHOLME, Joshua

Russian Academy of Sciences

Russian Federation

SUGA, Toshio

Tohoku University/JAMSTEC

Japan

SULISTYAWATI, Sulistyawati

Ahmad Dahlan University

Indonesia 
SULTAN, Maitham

Ministry of Science and Technology

Iraq

SUN, Jianqi

Chinese Academy of Sciences

China

SUN, Jun

Tianjin University of Science and Technology

China

\section{SUNDBY, Svein}

Institute of Marine Research

Norway

\section{SUNGHYUN, Nam}

Seoul National University

Republic of Korea

SURIANO, Zachary

University of Nebraska Omaha

USA

SUTTER, Johannes

Alfred Wegener Institute

Germany

SUTTON-GRIER, Ariana

The Nature Conservancy

USA

\section{SUTTON, Adrienne}

National Oceanographic and

Atmospheric Administraton

USA

\section{SUZUKI, Kazuyoshi \\ Japan Agency for Marine-Earth \\ Science and Technology \\ Japan}

SWART, Neil

Environment and Climate Change Canada

Canada

\section{TAILLARDAT, Pierre}

Université du Québec à Montréal

Canada

TAKAHASHI, Ken

Servicio Nacional de Meteorología

e Hidrología del Perú

Peru

TAKATA, Kumiko

National Institute for Environmental Studies Japan
TALLEY, Lynne

University of California San Diego

USA

TAMURA, Makoto

Ibaraki University

Japan

TANG, Malcolm

University of Malaya

Malaysia

TAQUI, Muhammad

Comsats University Islamabad

Pakistan

TARASOV, Lev

Memorial University of Newfoundland

Canada

TAYLOR, Patrick

National Aeronautics and Space Administration USA

TEDESCO, Letizia

Finnish Environment Institute

Finland

THAKUR, Praveen Kumar

Indian Institute of Remote Sensing

India

THALER, Thomas

University of Natural Resources

and Life Sciences

Austria

THIBERT, Emmanuel

Université Grenoble Alpes

France

THOMAS, Elizabeth

British Antarctic Survey

United Kingdom

THOMAS, Frank

University of the South Pacific

Fiji

\section{THOMSEN, Soeren}

Sorbonne University, IPSL, LOCEAN

France

THOR, Peter

Swedish Meteorological and

Hydrological Institute

Sweden
TIGNOR, Melinda

IPCC WGII TSU

Alfred Wegener Institute

Germany/USA

TINKER, Jonathan

Met Office Hadley Centre

United Kingdom

TIWARI, Pushp Raj

University of Hertfordshire

United Kingdom

TJERNSTRÖM, Michael

Stockholm University

Sweden

TOKARSKA, Katarzyna B.

Univerisity of Edinrbugh

United Kingdom

TOMANEK, Lars

California Polytechnic State University USA

TOTIN, Edmond

National University of Agriculture Benin

TOY, Suleyman

Atatürk University

Turkey

TREWIN, Blair

Australian Bureau of Meteorology Australia

TROVATO, Maria Rosa

University of Catania

Italy

TRUFFER, Martin

University of Alaska Fairbanks

USA

TSANI, Stella

International Centre for Research

on the Environment and the Economy

Greece

TURCO, Marco

Barcelona Supercomputing Center Spain

TURNER, Kate

University of Alaska Fairbanks

USA 


\section{ULTEE, Elizabeth}

Massachusetts Institute of Technology

USA

\author{
UOTILA, Petteri \\ University of Helsinki \\ Finland \\ UYSAL, Irfan \\ Ministry of Agriculture \& Forestry \\ Turkey
}

\section{VACHAUD, Georges \\ CNRS \\ France \\ VALLOT, Dorothée \\ Swedish Meteorological and \\ Hydrological Institute \\ Sweden}

VAN DE WAL, Roderik

Utrecht University

Netherlands

VAN DEN BROEKE, Michiel

Utrecht University

Netherlands

\section{VAN DEN HEUVEL, Floortje}

Ecole Polytechnique Federale de Lausanne

Switzerland

\section{VAN DER LAAN, Larissa}

Bavarian Academy of Sciences and Humanities Germany

\section{VAN DONGEN, Eef}

ETH Zurich

Switzerland

\section{VAN MEERBEECK, Cedric}

Caribbean Institute for

Meteorology and Hydrology

Barbados

\section{VAN WYCHEN, Wesley}

University of Waterloo

Canada

VAN YPERSELE, Jean-Pascal

Université Catholique de Louvain

Belgium

VARADE, Divyesh

Indian Institute of Technology Kanpur

India
VERFAILLIE, Deborah

Barcelona Supercomputing Center

Spain

VIEIRA, Gonçalo

Universidade de Lisboa

Portugal

VIJAY, Saurabh

Technical University of Denmark

Denmark

VINCENT, Christian

University of Grenoble/CNRS

France

VIVIAN, Christopher

Centre for Environment, Fisheries

and Aquaculture Science (Retired)

United Kingdom

VLADU, Iulian Florin

United Nations Framework

Convention on Climate Change

Germany

VON SCHUCKMANN, Karina

Mercator Ocean International

France

VOORTMAN, Hessel

Arcadis

Netherlands

VOUSDOUKAS, Michail

EC Joint Research Centre

Italy

WAGNER, Thomas

National Aeronautics and Space Administration USA

\section{WAGNER, Till}

University of North Carolina Wilmington USA

\section{WAGNON, Patrick}

IGE-IRD

France

WAHL, Thomas

University of Central Florida

USA

WALKER, Scott

Northwest Vista College

USA
WANG, Chunzai

Chinese Academy of Sciences

China

WANG, Dongxiao

South China Sea Institute of Oceanology

China

WANG, Feiteng

Chinese Academy of Sciences

China

WANG, Junye

Athabasca University

Canada

WANG, Pengling

China Meterological Administration

China

WANG, XIAOMING

Chinese Academy of Science

Australia

WANG, Xiujun

Beijing Normal University

China

WANG, Zhaomin

Hohai University

China

WARREN, Stephen

University of Washington

USA

WARRICK, Olivia

Red Cross Red Crescent Climate Centre

New Zealand

WATSON, Phil

US Coastal Education and

Research Foundation

Australia

WEATHERHEAD, Elizabeth

Jupiter Intelligence; University

of Colorado (Retired)

USA

WEBBER, Ben

University of East Anglia

United Kingdom

WEISSENBERGER, Sebastian

Université du Québec à Montréal

Canada 
WEN, Jiahong

Shanghai Normal University

China

WEPKING, Carl

Colorado State University

USA

\section{WESTER, Philippus \\ International Centre for Integrated \\ Mountain Development \\ Netherlands}

WEYER, Nora M.

IPCC WGII TSU

Alfred Wegener Institute

Germany

WHITE, Dave

Climate Change Truth Inc.

USA

\section{WHITE, Rehema \\ University of St Andrews \\ United Kingdom}

WHITEHOUSE, Pippa

Durham University

United Kingdom

WILLIAMS, Dee

United States Geological Survey

USA

\section{WILLIAMS, Emily}

University of California Santa Barbara USA

\section{WINBERG VON FRIESEN, Lisa}

IVL Swedish Environmental Research Institute Sweden

\section{WINIGER, Patrik}

Independent Expert

Netherlands

\section{WINKLER, Manuela}

University of Natural Resources

and Life Sciences

Austria

WOLFF, Eric

University of Cambridge

United Kingdom
WONG, Poh Poh

University of Adelaide

Singapore

WOOD, Thomas

Retired

USA

WRIGHT, Jeneva

East Carolina University

USA

WU, Bingyi

Fudan University

China

WU, Mengxi

Brown University

USA

\section{WU, Renguang}

Chinese Academy of Sciences

China

\section{WU, Shaohong}

Chinese Academy of Sciences

China

WUITE, Jan

Environmental Earth Observation

Information Technology $\mathrm{GmbH}$

Austria

YAMANOUCHI, Takashi

National Institute of Polar Research

Japan

YANG, Handa

University of California

USA

YANG, Y. Jeffrey

United States Environmental Protection Agency

USA

\section{YATES, Katherine \\ University of Salford \\ United Kingdom}

YETTELLA, Vineel

University of Colorado Boulder

USA

YIN, Baoshu

Chinese Academy of Sciences

China
YIN, Yixing

Nanjing University of Information

Science and Technology

China

YOUNG, Gillian

British Antarctic Survey

United Kingdom

YOUNG, Tun Jan

Taroko National Park

United Kingdom

YOUNGFLESH, Casey

Stony Brook University

USA

YUMRUKTEPE, Veli Caglar

Nansen Environmental and

Remore Sensing Center

Norway

ZAELKE, Durwood Institute for Governance and Sustainable Development USA

ZAFAR, Qudsia

Global Change Impact Studies Centre Pakistan

ZAITON IBRAHIM, Zelina

Malaysia

ZAREIAN, Mohammad Javad Ministry of Energy

Iran

ZARGARLELLAHI, Hanieh Geological Survey of Iran Iran

ZEKOLLARI, Harry

ETH Zürich

Switzerland

ZHAI, Panmao

IPCC WGI Co-Chair

Meteorological Administration

China

ZHANG, Fan

Chinese Academy of Sciences

China 
ZHANG, Rui

University of British Columbia

Canada

ZHOU, Botao

Nanjing University of Information

Science and Technology

China

ZINKE, Jens

Freie Universität Berlin

Germany

ZIVERI, Patrizia

ICREA-ICTA UAB

Spain

ZIVIAN, Anna

Ocean Conservancy

USA

\section{ZOLKOS, Scott}

University of Alberta

USA

ZUO, Juncheng

Zhejiang Ocean University

China 